\title{
A RARE CASE OF A FUNCTIONAL NON COMMUNICATING HORN IN UNICORNUATE UTERUS IN POSTMENARCHEAL ADOLESCENT GIRL
}

\author{
Vijayalaxmi R. Gobbur ${ }^{1}$, Shreedevi Kori ${ }^{2}$
}

\section{HOW TO CITE THIS ARTICLE:}

Vijayalaxmi R. Gobbur, Shreedevi Kori. "A Rare Case of a Functional Non communicating Horn in Unicornuate Uterus in Postmenarchal Adolescent Girl". Journal of Evolution of Medical and Dental Sciences 2014; Vol. 3, Issue 25, June 23; Page: 6920-6922, DOI: $10.14260 /$ jemds/2014/2839

ABSTRACT: A 15-year-old girl presented with acute abdomen. On exploratory laparotomy she was found to have a right functional rudimentary horn of the uterus. We report a case of unicornuate uterus with functioning rudimentary horn. We here in highlight that mullerian duct anomalies should be considered in the differential diagnosis of acute abdomen even in normally menstruating girls. Unicornuate uterus with a rudimentary horn is susceptible to many gynecologic and obstetric complications.

Hematometra, chronic pelvic pain, endometriosis, infertility are some of the complaints in women with unicornuate uterus.

KEYWORDS: Unicornuate uterus, Rudimentary horn, Hematometra.

MESHTERMS: MDA, Unicornuate uterus, Rudimentary horn, Hematometra.

INTRODUCTION: Mullerian duct anomalies (MDA) are a miscellaneous group of entities that result from non-development, defective vertical or lateral fusion, or resorption failure of mullerian (paramesonephric) ducts ${ }^{[1]}$.Although majority are asymptomatic, the reported prevalence of MDAs ranges between $0.4 \%$ (in women who present for non-obstetric USG) and 8-10\% in women with recurrent pregnancy loss. ${ }^{[2]}$ Average incidence of MDA is $0.2-1.4 \%$.

But occurrence of functional non-communicating horn is rare. We are reporting such a fascinating case of functional non-communicating horn in unicornuate uterus in a young girl presenting as acute abdomen.

CASE REPORT: Unmarried 15 yrs old girl came with complaints of pain abdomen since 4 days to OPD. Pain was spasmodic in nature and associated with vomiting. Pain not associated with any aggravating and relieving factors.

She had attained menarche 3 yrs back and had regular cycles of 3-4days flow for every 28-30 days but associated with severe dysmenorrhea for 2-3 days. LMP: 15days back and had similar complaints $1 \mathrm{yr}$ back and had underwent appendectomy for same complaints.

O/E: PR: 90bpm, BP: 110/70 mmHg, Afebrile.

$\mathrm{P} / \mathrm{A}$ Examination - soft and tenderness present in suprapubic region and right iliac fossa, appendectomy scar healthy.

USG showed uterine size normal, $5 \times 5 \mathrm{cms}$ spherical mass in right cornu of uterus and fluid in mass with both ovaries normal.

A provisional diagnosis of? Bicornuate uterus and? Cornual fibroid was made. Patient was posted for laparotomy. At laparotomy, Hematometra present in non-communicating horn on right side of unicornuate uterus. Incision made over the mass and chocolate colored fluid drained (20$30 \mathrm{ml}$ ).Uterine cavity visualized- Non communicating horn of unicornuate uterus confirmed. 
Adhesions and endometriotic deposits were present in pouch of douglas, right tube and ovary. Adhesions lysed and endometriotic deposits coagulated. Left tube and ovary were found normal. Rudimentary horn excised along with right sided salphingoopherectomy. Patient recovered well postoperatively. The patient had attended our outpatient department for follow-up and had no complaints.

DISCUSSION: MDA are some of the fascinating disorders encountered by obstetricians and gynecologists. They are frequently associated with renal and axial system (skeletal) abnormalities. Unicornuate uterus with a rudimentary horn is rare with incidence of $0.06 \% .{ }^{[3]}$ In $83 \%$ of the cases, the rudimentary horn is non-communicating.[4]

Early identification of MDAs helps to avoid prolonged symptomatic periods in young adolescents and the complications that may subsequently arise, such as hydronephrosis (mostly in obstructed anomalies), endometriosis and infertility. As 30\% of MDA's are associated with renal tract anomalies, investigation for renal function is imperative ${ }^{[5]}$

Rudimentary horn can be functional (endometrial layer +) or nonfunctional (endometrial layer absent).

USG is the initial imaging modality in the evaluation of these anomalies, however to identify the uterine remnant and to identify normal uterus MRI is a complement. MRI differentiates between uterine agenesis and hypoplasia (both in sagittal sections).

Management includes counseling which is very important in MDA's for both patient and her parents. In case of non-communicating functional rudimentary horn of unicornuate uterus manifestations are at menarche either dysmenorrheal and pelvic pain present or they may go undiagnosed as less reproductive problems. Resection of the rudimentary horn should be considered such patients if left untreated have more chances of ectopic pregnancies, endometriosis and infertility.

Accurate diagnosis is essential since management approach varies on various anomalies.

CONCLUSION: Acute abdomen should be thoroughly evaluated especially in adolescents with relevant investigations keeping in mind the rare possibility of mullerian anomaly. Early diagnosis and prompt treatment is recommended in these cases to avoid future gynecological and obstetrical complications. The right step at the right moment aided with maximal suspicion and investigation and minimal access surgery is the preferred mode of management here.

\section{REFERENCES:}

1. Laufer MR, Goldstein DP, Hendren W H. Structural abnormalities of female reproductive tract. Paediatric and adolescent gynaecology. $5^{\text {th }}$ edition. Boston, Mars: Lippincott Williams and wilkins, 2005; 362-416.

2. Byrne J, Nussbaum-Blask- A, Taylor WS. Prevalence of mullerian duct anamolies detected at USG. Am J med Genet 2000; 9-12

3. Raga F, Bauset C, Remohi J, Bonilla-Musoles F, Simón C, Pellicer A. Reproductive impact of congenital mullerian anomalies. Hum Reprod 1997; 12: 2277-81

4. Heinonen PK. Unicornuate uterus and rudimentary horn. Fertil Steril 1997; 68: 224-30. 


\section{CASE REPORT}

5. Lis, Qayyum A, Coakley FV, Hricak H. Association of renal agenesis and mullerian duct anamolies. Jcomput assisted Tomography 2000; 24(6): 829-834.

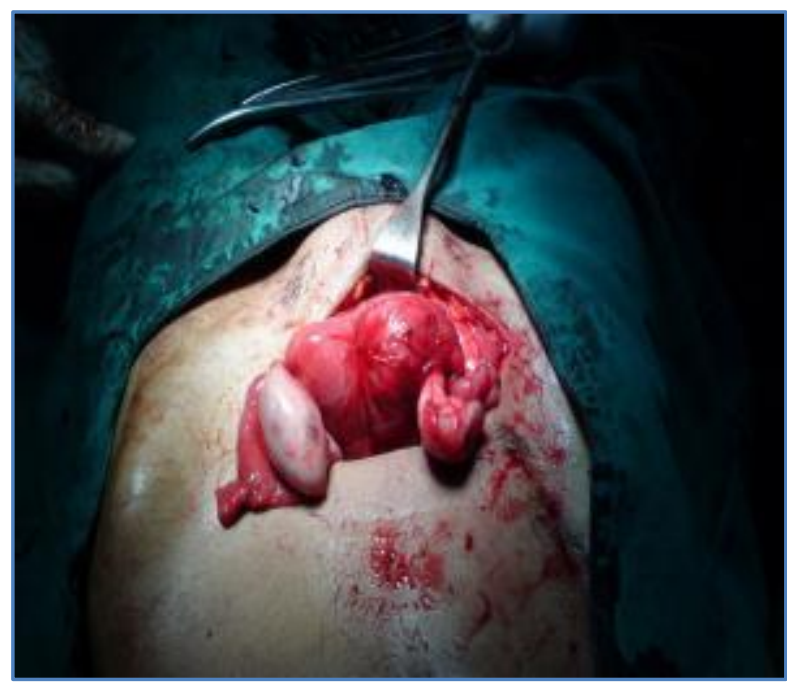

Non communicating functional Rudimentary horn in unicornuate uerus

\section{AUTHORS:}

1. Vijayalaxmi R. Gobbur

2. Shreedevi Kori

\section{PARTICULARS OF CONTRIBUTORS:}

1. Professor, Department of Obstetrics and Gynaecology, BLDE University's Shri B. M. Patil Medical College and Research Center, Bijapur.

2. Senior Resident, Department of Obstetrics and Gynaecology, BLDE University's Shri B. M. Patil Medical College and Research Center, Bijapur.



Rudimentary horn excised

\section{NAME ADDRESS EMAIL ID OF THE CORRESPONDING AUTHOR:}

Dr. Shreedevi Kori, Senior Resident, Department of Obstetrics and Gynaecology, Shri B. M. Patil Medical College and Research Center, Bijapur-586103.

Email: shreedevi_Kori@yahoo.com

Date of Submission: 08/06/2014. Date of Peer Review: 09/06/2014. Date of Acceptance: 16/06/2014. Date of Publishing: 19/06/2014. 